\title{
Numerical Study of Entropy Generation with Nonlinear Thermal Radiation on Magnetohydrodynamics non-Newtonian Nanofluid Through a Porous Shrinking Sheet
}

\author{
M. M. Bhatti ${ }^{1 *}$, T. Abbas ${ }^{2}$, and M. M. Rashidi ${ }^{3,4}$ \\ ${ }^{1}$ Shanghai Institute of Applied Mathematics and Mechanics, Shanghai University, Shanghai 200072, China \\ ${ }^{2}$ Department of Mathematics, Quaid-I-Azam University, Islamabad 44000, Pakistan \\ ${ }^{3}$ Shanghai Key Lab of Vehicle Aerodynamics and Vehicle Thermal Management Systems, Tongji University, Shanghai 201804, China \\ ${ }^{4}$ ENN-Tongji Clean Energy Institute of Advanced Studies, Shanghai 200072, Tongji University, China
}

(Received 20 April 2016, Received in final form 15 June 2016, Accepted 16 June 2016)

\begin{abstract}
In this article, entropy generation on MHD Williamson nanofluid over a porous shrinking sheet has been analyzed. Nonlinear thermal radiation and chemical reaction effects are also taken into account with the help of energy and concentration equation. The fluid is electrically conducting by an external applied magnetic field while the induced magnetic field is assumed to be negligible due to small magnetic Reynolds number. The governing equations are first converted into the dimensionless expression with the help of similarity transformation variables. The solution of the highly nonlinear coupled ordinary differential equation has been obtained with the combination of Successive linearization method (SLM) and Chebyshev spectral collocation method. Influence of all the emerging parameters on entropy profile, temperature profile and concentration profile are plotted and discussed. Nusselt number and Sherwood number are also computed and analyzed. It is observed that entropy profile increases for all the physical parameters. Moreover, it is found that when the fluid depicts non-Newtonian (Williamson fluid) behavior then it causes reduction in the velocity of fluid, however, non-Newtonian behavior enhances the temperature and nanoparticle concentration profile.
\end{abstract}

Keywords : nanofluid, entropy generation, thermal radiation, shrinking sheet, SLM

\section{Introduction}

During the past years, nanofluid has received a remarkable attention by various researchers due to its number of application in industry. The homogenous combination of base fluid and ultrafine nanoparticles are known as nanofluid. The nanoparticles are made of various metals or non-metals such as aluminum (Al), copper $(\mathrm{Cu})$, Silver $(\mathrm{Ag})$, graphite, carbon nanotubes respectively, and the base fluid which includes oil or ethylene glycol and water. The suspension of nanoparticles is very much helpful to increase the heat transfer performance and thermal conductivity. This technique is the most powerful and modern to enhance the coefficient of heat transfer. In solid metals, the thermal conductivity is very much higher as compared to the base fluids. Nanofluids have numerous applications in industrial engineering and techno-

CThe Korean Magnetics Society. All rights reserved.

*Corresponding author: Tel: +13162146836

Fax:+13162146836, e-mail: muhammad09@shu.edu logical applications. Such type of applications includes heat exchanger, vehicle cooling, nuclear reactor, cooling of electronic devices and vehicle thermal management respectively. The magneto nanofluids are also very much helpful in magnetic drug targeting in cancer diseases, hyperthermia, wound treatments, removal of blockage in the arteries, magnetic resonance imaging (MRI) etc. Choi [1] was the first who introduced the concept of nanofluid and described that the embedding of nanoparticles in a base fluid is very much helpful to increase the thermal properties of base fluids. Xuan and $\mathrm{Li}$ [2] investigated on convective heat transfer and flow properties of nanofluids. Buongiorno [3] introduced a new mathematical model to analyze the thermal features of base fluids. He utilized the thermophoresis and Brownian motion to enhance the thermal features of base fluids. Later, various researchers investigated the nanofluid problems with differential geometrical aspects. For instance, Khan and pop [4] analyzed the boundary layer flow of nanofluid past a stretching sheet with the help of Buongiorno [3] model. Mustafa et al. [5] studied the stagnation point flow of nanofluid over 
a stretching sheet. Some more pertinent studies on the said topic can be found from the refs. [6-10] and several therein.

Entropy generation is a function that measures the level of irreversibilities in a process. According to the thermodynamics approach, the entropy generation minimization technique is applied to enhance thermal engineering devices for its better efficiency. During the occupancy of irreversibilities, the performance of engineering equipment decreases. According to the recent analysis [11-13], it is found that second law of thermodynamic is more efficient and powerful as compared to the first law of thermodynamics. During the past years, various authors have utilized the application of second law of thermodynamics during the manufacturing of thermal engineering systems. Oztop and Salem [14] explored the entropy generation in mixed and natural convection heat transfer for energy systems. Rashidi et al. [15] analyzed the Entropy generation on MHD nanofluid flow over a rotating porous disk. Abolbashari et al. [16] investigated the entropy generation on MHD nanofluid over a permeable stretching surface. Later, Abolbashari et al. [17] analyzed analytically the entropy generation of non-Newtonian Casson nanofluid over a stretching surface. Some more studies on entropy generation can be found from refs. [18-24].

On the other hand, the study of boundary layer flow of Newtonian and non-Newtonian nanofluid over stretching/ shrinking surface is also very important in different engineering and industrial applications. In particular, such type fluids are applicable in nuclear and chemical industry, foodstuff, material processing, oil engineering reservoirs, production of paper, glass fiber, wire drawing, drawing of plastic films, hot rolling and extrusion of plastic sheets respectively. Non-Newtonian fluid depicts various behaviors which cannot be analyzed using single relationship. NonNewtonian fluids are divided into three groups such as integral type, rate type, and differential type. Abbas et al. [25] analyzed numerically the stagnation point flow of Casson fluid over stretching/shrinking sheet under the influence of thermal radiation and chemical reaction. Khan et al. [26] investigated the three-dimensional nanofluid flow over the nonlinear stretching sheet with the application of solar energy. Rashidi et al. [27] analyzed the MHD viscoelastic fluid flow over a porous wedge with heat transfer and thermal radiation. Rashidi et al. [28] examined the influence of buoyancy effect on nanofluid flow over a stretching sheet with thermal radiation and magnetohydrodynamics. Some relevant studies on the said topic are available in the references [29-34].

With the above analysis in mind, the aim of present study is to analyze the entropy generation on MHD William- son nanofluid over a porous shrinking sheet. The governing flow problem is based on momentum equation, energy equation and nanoparticle concentration which are simplified with the help of similarity transformation variables. The reduced ordinary coupled differential equations are solved numerically with the help of Successive linearization method (SLM) and Chebyshev spectral collocation method [37-39]. The present methodology is better and converges more rapidly as compared to other computational methods $[22,40]$. A suitable initial guess is selected that satisfies the governing boundary conditions and the governing equations are solved iteratively. This paper is summarized as follows: after the introduction in Sec. (1), Sec. (2) based on the mathematical formulation of the problem, Sec. (3) deals with the physical quantities, Sec. (4) describes the methodology of the problem, Sec. (5) characterize the entropy generation analysis and finally Sec. (6) devoted to numerical results and discussion.

\section{Mathematical Formulation}

Consider the MHD boundary layer flow over a porous shrinking surface near a stagnation point at $y=0$. The MHD flow occurs in the domain at $y>0$ The fluid is electrically conducting by an external magnetic field while the induced magnetic is assumed to be zero. Cartesian coordinate is chosen in a way such that $x$-axis is considered along the direction of the sheet whereas $y$-axis is considered along normal to it. Suppose that $C_{w}$ be the nano particle fraction at the sheet while the temperature and nano-particle fraction at infinity is $T_{\infty}$ and $C_{\infty}$. The velocity of the sheet is considered along $x$-direction $\tilde{u}_{w}=a x$

The governing equations of Williamson nanofluid model can be written as [39]

$$
\frac{\partial \tilde{u}}{\partial x}+\frac{\partial \tilde{v}}{\partial y}=0
$$

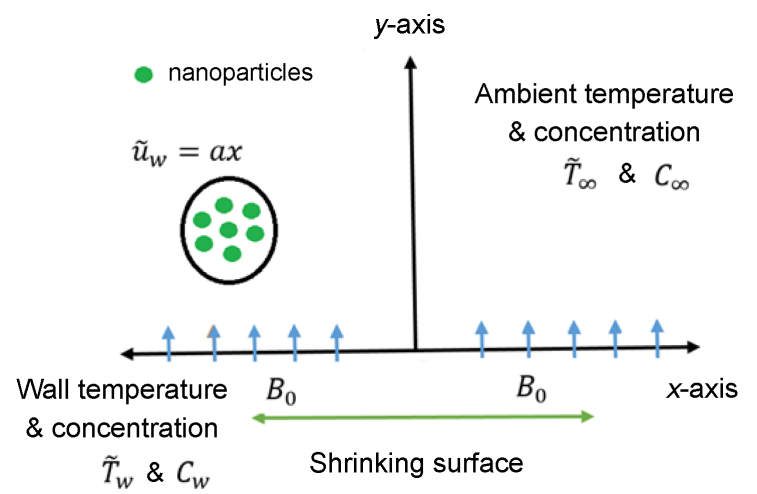

Fig. 1. (Color online) Geometry of the problem. 


$$
\begin{aligned}
& \tilde{u} \frac{\partial \tilde{u}}{\partial x}+\tilde{v} \frac{\partial \tilde{v}}{\partial y}= v \frac{\partial^{2} \tilde{u}}{\partial y^{2}}+\sqrt{2} \bar{\Gamma} \frac{\partial \tilde{u}}{\partial y} \frac{\partial^{2} \tilde{u}}{\partial y^{2}}+\tilde{u}_{e} \frac{\partial \tilde{u}_{e}}{\partial x} \\
&+\left(\frac{v}{\bar{k}}+\frac{\sigma B_{0}^{2}}{\rho}\right)\left(\tilde{u}_{e}-\tilde{u}\right), \\
& \tilde{u} \frac{\partial \tilde{T}}{\partial x}+\tilde{v} \frac{\partial \tilde{T}}{\partial y}= \bar{\alpha} \frac{\partial^{2} \tilde{T}}{\partial y^{2}}+\tau\left(D_{B} \frac{\partial C}{\partial y} \frac{\partial \tilde{T}}{\partial y}+\frac{D_{T}}{T_{\infty}}\left(\frac{\partial \tilde{T}}{\partial y}\right)^{2}\right)-\frac{1}{\rho c_{p}} \frac{\partial q_{r}}{\partial y}, \\
& \tilde{u} \frac{\partial C}{\partial x}+\tilde{v} \frac{\partial C}{\partial y}=D_{B} \frac{\partial^{2} C}{\partial y^{2}}+\frac{D_{T}}{T_{\infty}} \frac{\partial^{2} \tilde{T}}{\partial y^{2}}-K\left(C-C_{\infty}\right) .
\end{aligned}
$$

The nonlinear radiative heat flux can be written as

$$
q_{r}=-\frac{4 \bar{\sigma}}{3 \bar{k}} \frac{\partial \tilde{T}^{4}}{\partial y}=-\frac{16 \bar{\sigma} \tilde{T}^{3}}{3 \bar{k}} \frac{\partial \tilde{T}}{\partial y}
$$

and their respective boundary conditions are

$$
\begin{aligned}
& \tilde{u}=u_{w}, v=v_{w}, \tilde{T}=\tilde{T}_{w}, C=C_{w} \text { at } y=0, \\
& \tilde{u}=\tilde{u}_{e}, \tilde{v}=0, \tilde{T} \rightarrow \tilde{T}_{\infty}, C \rightarrow C_{\infty} \text { as } y \rightarrow \infty .
\end{aligned}
$$

The steam function satisfying Eq. (1) are defined as $\tilde{u}=\partial \varphi / \partial y$ and $\tilde{v}=\partial \varphi / \partial x$. Defining the following similarity transformation variables

$$
\begin{gathered}
\zeta=\sqrt{\frac{\tilde{u}_{w}}{v x}} y, \tilde{u}=\tilde{u}_{w} f^{\prime}(\zeta), \tilde{v}=-\sqrt{\frac{v \tilde{u}_{w}}{x}} f(\zeta), \\
\theta=\frac{\tilde{T}-\tilde{T}_{\infty}}{\tilde{T}_{w}-\tilde{T}_{\infty}}, \phi=\frac{C-C_{\infty}}{C_{w}-C_{\infty}},
\end{gathered}
$$

and using Eq. (8) in to Eq. (7) and Eq. (3), we get

$$
\begin{aligned}
& f^{\prime \prime \prime}+1+\lambda f^{\prime \prime} f^{\prime \prime \prime}-f^{\prime 2}+f f^{\prime \prime}+\Gamma\left(1-f^{\prime}\right)=0, \\
& \left(\frac{1}{P_{r}}+\frac{4}{3} N_{r}\right) \theta^{\prime \prime}+f \theta^{\prime}+N_{b} \theta^{\prime} \phi^{\prime}+N_{t}\left(\theta^{\prime}\right)^{2}=0, \\
& \phi^{\prime \prime}+L_{e} f \phi^{\prime}+\frac{N_{t}}{N_{b}} \theta^{\prime \prime}-\gamma \Phi=0 .
\end{aligned}
$$

Their corresponding boundary conditions are

$$
\begin{aligned}
& f(0)=S, f^{\prime}(0)=\alpha, f^{\prime}(\infty)=1, \\
& \theta(0)=1, \theta(\infty)=0, \\
& \phi(0)=1, \phi(\infty)=0,
\end{aligned}
$$

where $P_{r}=v / \bar{\alpha}, k=v / \bar{k}, M=B_{0}^{2} \sigma / c \rho, L_{e}=\frac{v}{D_{B}}, N_{b}=$ $\frac{\tau D_{B}\left(C_{w}-C_{\infty}\right)}{v}, N_{t}=\frac{\tau D_{T}\left(\tilde{T}_{w}-\tilde{T}_{\infty}\right)}{T_{\infty} v}, N_{r}=\frac{4 \bar{\sigma} \tilde{T}^{3}}{\rho c_{p} \bar{k}}, \Gamma=M+k$.

\section{Physical Quantities of Interest}

The physical quantities of interest for the governing flow problem are local Nusselt number and local Sherwood number which can be written as

$$
N u_{x}=\frac{x q_{w}}{\kappa\left(\tilde{T}_{w}-\tilde{T}_{\infty}\right)}, S h_{x}=\frac{x q_{m}}{D_{B}\left(C_{w}-C_{\infty}\right)},
$$

where $q_{w}$ and $q_{m}$ are described as

$$
q_{w}=-\kappa\left(\bar{\alpha}+\frac{16 \bar{\sigma} \tilde{T}^{3}}{3 \bar{k}}\right)\left(\frac{\partial \tilde{T}}{\partial y}\right)_{y=0_{y=0}}, q_{m}=D_{B}\left(-\frac{\partial C}{\partial y}\right)_{y=0} .
$$

With the help of dimensionless transformation in Eq. (8), we have

$$
N u_{r}=\frac{N u_{x}}{\operatorname{Re}_{x}^{\frac{1}{2}}}=-\left(1+\frac{4}{3} N_{r}\right) \theta^{\prime}(0), S h_{r}=\frac{S h_{x}}{\operatorname{Re}_{x}^{\frac{1}{2}}}=-\phi^{\prime}(0),
$$

where $S h_{r}$ and $N u_{r}$ are the dimensionless Sherwood number and local Nusselt number, respectively and $\operatorname{Re}_{x}=\tilde{u}_{w} x / v$ is the local Reynolds number.

\section{Numerical Method}

We apply the Successive linearization method to Eq. (9) with their boundary conditions in Eq. (12), by setting [41]

$$
f(\zeta)=f_{I}(\zeta)+\Sigma_{N=0}^{I-1} f_{N}(\zeta),(I=1,2,3, \ldots),
$$

where $f_{I}$ are unknown functions which are obtained by iteratively solving the linearized version of the governing equation and assuming that $f_{I}(0 \leq N \leq I-1)$ are known from previous iterations. Our algorithm starts with an initial approximation $f_{0}$ which satisfy the given boundary conditions in Eq. (13) according to SLM. The suitable initial guess for the governing flow problem is

$$
f_{0}=-1+\zeta+\alpha+S+\frac{1-\alpha}{e^{\zeta}} .
$$

We write the equation in general form as

$$
\mathbf{L}\left(f, f^{\prime}, f^{\prime \prime}, f^{\prime \prime \prime}\right)+\mathbf{N}\left(f, f^{\prime}, f^{\prime \prime}, f^{\prime \prime \prime}\right)=0,
$$

where

$$
\mathbf{L}\left(f, f^{\prime}, f^{\prime \prime}, f^{\prime \prime \prime}\right)=f^{\prime \prime \prime},
$$

and

$$
\mathbf{N}\left(f, f^{\prime}, f^{\prime \prime}, f^{\prime \prime \prime}\right)=f f^{\prime \prime}+1-f^{\prime 2}+\Gamma\left(1-f^{\prime}\right)+W e f^{\prime \prime} f^{\prime \prime \prime},
$$

where $\mathbf{L}$ and $\mathbf{N}$ are the linear and non-linear part of Eq. (9). By substituting Eq. (18) in Eq. (9) and taking the linear terms only, we get

$$
f_{I}^{\prime \prime \prime}+A_{0, I-1} f_{I}^{\prime \prime}+A_{1, I-1} f_{I}^{\prime}+A_{2, I-1} f_{I}=\mathbf{r}_{I-1},
$$

the corresponding boundary conditions becomes

$$
f_{I}(0)=0, f_{I}^{\prime}(0)=0, f_{I}^{\prime}(\infty)=0 .
$$

We solve Eq. (23) numerically by a well-known method namely Chebyshev spectral collocation method. For 
numerical implementation, the physical region $[0, \infty)$ is truncated to $[0, \Gamma]$ we can take $\Gamma$ to be sufficient large. With the help of following transformations this region is further transformed in to $[-1,1]$, we have

$$
\Omega=-1+\frac{2 \zeta}{\Gamma} \text {. }
$$

We define the following discretization between the interval $[-1,1]$. Now, we can apply Gause-Lobatto collocation points to define the nodes between $[-1,1]$ by

$$
\Omega_{J}=\cos \frac{\pi J}{N}, \quad(J=0,1,2,3, \ldots N),
$$

with $(N+1)$ number of collocation points. Chebyshev spectral collocation method based on the concept of differentiation matrix D. This differentiation matrix maps a vector of the function values $\mathbf{G}=\left[f\left(\Omega_{0}\right), \ldots, f\left(\Omega_{N}\right)\right]^{T}$ the collocation points to a vector $\mathbf{G}^{\prime}$ is defined as

$$
\mathbf{G}^{\prime}=\Sigma_{K=0}^{N} \mathbf{D}_{K J} f\left(\Omega_{K}\right)=\mathbf{D G},
$$

the derivative of $p$ order for the function $f(\zeta)$ can be written as

$$
f^{p}(\Omega)=\mathbf{D}^{p} \mathbf{G} .
$$

The entries of matrix $\mathbf{D}$ can be computed by the method proposed by Bhatti et al. [41]. Now, applying the spectral method, with derivative matrices on linearized equation Eq. (23) and Eq. (24), we get the following linearized matrix system

$$
\mathbf{A}_{I-1} \mathbf{G}_{I}=\mathbf{R}_{I-1},
$$

the boundary conditions takes the following form

$$
\begin{aligned}
f_{I}\left(\Omega_{N}\right)= & 0, \sum_{K=0}^{N} \mathbf{D}_{N K} f_{I}\left(\Omega_{K}\right)=0, \Sigma_{K=0}^{N} \mathbf{D}_{0 K} f_{I}\left(\Omega_{K}\right)=0, \\
& \sum_{K=0}^{N} \mathbf{D}_{0 K}^{2} f_{I}\left(\Omega_{K}\right)=0,
\end{aligned}
$$

where

$$
\mathbf{A}_{I-1}=\mathbf{D}^{3}+A_{0, I-1} \mathbf{D}^{2}+A_{1, I-1} \mathbf{D}+A_{2, I-1} .
$$

In the above equation $A_{s, I-1}(s=0,1, \ldots 3)$ are $(N+1) \times$ $(N+1)$ diagonal matrices with $A_{s, I-1}\left(\Omega_{J}\right)$ on the main diagonal and

$$
\mathbf{G}_{I}=f_{I}\left(\Omega_{J}\right), \mathbf{R}_{I}=\mathbf{r}_{I}\left(\Omega_{J}\right) .(J=0,1,2,3, \ldots N) .
$$

After employing Eq. (31) on the solutions for $f_{I}$ are obtained by solving iteratively Eq. (30). We obtain the solution for $f(\zeta)$ from solving Eq. (31) and now Eq. (10) and Eq. (11) are now linear therefore, we will apply Chebyshev pseudo-spectral method directly, we get

$$
\mathbf{B H}=\mathbf{S},
$$

with their corresponding boundary conditions boundary conditions

$$
\begin{aligned}
& \theta\left(\Omega_{N}\right)=1, \theta\left(\Omega_{0}\right)=0, \\
& \phi\left(\Omega_{N}\right)=1, \phi\left(\Omega_{0}\right)=0,
\end{aligned}
$$

where $\mathbf{H}=\left(\theta\left(\Omega_{J}\right), \phi\left(\Omega_{J}\right)\right), \mathbf{B}$ is the set of linear coupled equation of temperature and nanoparticle concentration, $\mathbf{S}$ is a vector of zeros, and all vectors in Eq. (33) are converted to diagonal matrix. We imposed the boundary conditions in Eq. (34) and Eq. (35) on the first and last rows of $\mathbf{B}$ and $\mathbf{S}$ respectively.

\section{Entropy Generation Analysis}

The volumetric entropy generation of the Williamson nanofluid is given by [37-39]

$$
\begin{aligned}
& S_{\text {gen }}^{\prime \prime \prime}=\frac{\kappa}{\tilde{T}_{\infty}^{2}}\left[\left(\frac{\partial \tilde{T}}{\partial y}\right)^{2}+\frac{16 \bar{\sigma} \tilde{T}^{3}}{3 \bar{k}}\left(\frac{\partial \tilde{T}}{\partial y}\right)^{2}\right]+\frac{\mu}{\tilde{T}_{\infty}}\left(\left(\frac{\partial \tilde{u}}{\partial y}\right)^{2}+\frac{\bar{\Gamma}}{\sqrt{2}}\left(\frac{\partial \tilde{u}}{\partial y}\right)^{3}\right) \\
& +\frac{R D}{C_{\infty}}\left(\frac{\partial C}{\partial y}\right)^{2}+\frac{\sigma B_{0}^{2}}{\tilde{T}_{\infty}} \tilde{u}^{2}+\frac{v \tilde{u}^{2}}{\tilde{T}_{\infty} \tilde{k}}+\frac{R D}{\tilde{T}_{\infty}}\left(\frac{\partial \tilde{T}}{\partial y} \frac{\partial C}{\partial y}+\frac{\partial C}{\partial x} \frac{\partial \tilde{T}}{\partial x}\right)
\end{aligned}
$$

In the above equation, the entropy generation consists of three effects, (i) conduction effect (also known as heat transfer irreversibility, (HTI)); (ii) fluid friction irreversibility (FFI) and (iii) diffusion (also known as diffusive irreversibility, (DI)). The characteristics entropy generation can be written as

$$
S_{0}^{\prime \prime \prime}=\frac{\kappa(\Delta T)^{2}}{L^{2} \tilde{T}_{\infty}^{2}}
$$

With the help of Eq. (8), the entropy generation in dimensionless form can be written as

$$
\begin{aligned}
N_{G}= & \frac{s_{g e n}^{\prime \prime \prime}}{s_{0}^{\prime \prime \prime}}=\operatorname{Re}\left(1+\frac{4}{3} N_{r}\right) \theta^{\prime 2}(\zeta)+\frac{\operatorname{Re} B_{r}}{\Omega}\left(f^{\prime \prime 2}(\zeta)+W e f^{\prime \prime}(\zeta)\right) \\
& +\frac{\operatorname{Re} B_{r}}{\Omega} \Gamma f^{\prime 2}(\zeta)+\operatorname{Re} \lambda_{1}(\zeta)\left(\frac{\chi}{\Omega}\right)^{2} \phi^{\prime 2}(\zeta) \\
& +\operatorname{Re} \lambda_{1}\left(\frac{\chi}{\Omega}\right) \theta^{\prime}(\zeta) \phi^{\prime}(\zeta)
\end{aligned}
$$

These number are given in the following form

$$
\operatorname{Re}=\frac{\tilde{u}_{L} L^{2}}{v}, B_{r}=\frac{\mu \tilde{u}_{w}^{2}}{\kappa \Delta T}, \Omega=\frac{\Delta T}{\tilde{T}_{\infty}}, \chi=\frac{\Delta C}{C_{\infty}}, \lambda_{1}=\frac{R D C_{\infty}}{\kappa} .
$$

\section{Results and Discussion}

In this section, the effects of various emerging parameters such as Prandtl number, Brownian motion parameter, thermophoresis parameter, radiation parameter, chemical reaction parameter, magnetic parameter, porosity parameter, Lewis number, Brinkmann number and Reynolds 
Table 1. Numerical values of reduced Nusselt number $\left(N u_{r}\right)$ for various values of $\operatorname{Pr}, N_{r}, N_{b}$ and $N_{t}$.

\begin{tabular}{ccccc}
\hline \hline $\operatorname{Pr}$ & $N_{r}$ & $N_{b}$ & $N_{t}$ & $N u_{r}$ \\
\hline 2 & 1 & 0.5 & 0.5 & 1.0715 \\
4 & & & & 1.1214 \\
6 & & & & 1.1403 \\
& 0.5 & & & 0.7883 \\
& 1.5 & & & 1.1868 \\
& 2.0 & & & 1.3708 \\
& & 0.6 & & 0.9822 \\
& & 1.0 & & 0.9386 \\
& & 1.5 & & 0.8957 \\
& & & 0.4 & 1.0082 \\
& & & 0.9 & 0.9486 \\
& & & 1.4 & 0.9034 \\
\hline
\end{tabular}

Table 2. Numerical values of reduced Sherwood number $\left(S h_{r}\right)$ for various values of $\gamma, L_{e}, N_{b}$ and $N_{t}$.

\begin{tabular}{ccccc}
\hline \hline$\gamma$ & $L_{e}$ & $N_{b}$ & $N_{t}$ & $S h_{r}$ \\
\hline 0.1 & 0.5 & 0.5 & 0.5 & 0.4510 \\
0.5 & & & & 0.6377 \\
1.0 & & & & 0.8215 \\
& 0.6 & & & 0.4802 \\
& 1.0 & & & 0.6001 \\
& 1.5 & & & 0.7544 \\
& & 0.6 & & 0.4690 \\
& & 1.0 & & 0.5166 \\
& & 1.5 & & 0.5492 \\
& & & 0.6 & 0.4329 \\
& & & 1.0 & 0.3845 \\
& & & 1.5 & 0.3510 \\
\hline
\end{tabular}

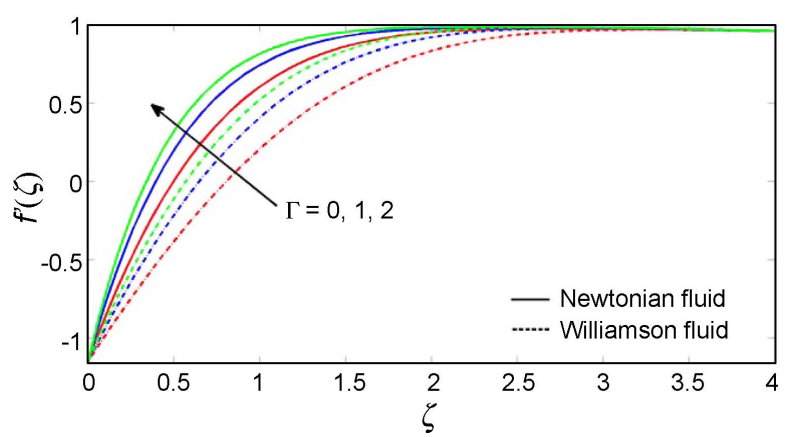

Fig. 2. (Color online) Velocity profile for different values of $\Gamma$ when $\operatorname{Pr}=1, N_{r}=0.5, \alpha=-1.15, \gamma=0.5, N_{b}=0.2, N_{t}=0.2$, $L_{e}=0.5$.

number are plotted for velocity, temperature, nanoparticle concentration and entropy profile. For this purpose, Fig. 2 to Fig. 14 are sketched. Furthermore, all the graphical results are demonstrated for Newtonian and Williamson fluid. Table 1 and Table 2 show the numerical computation

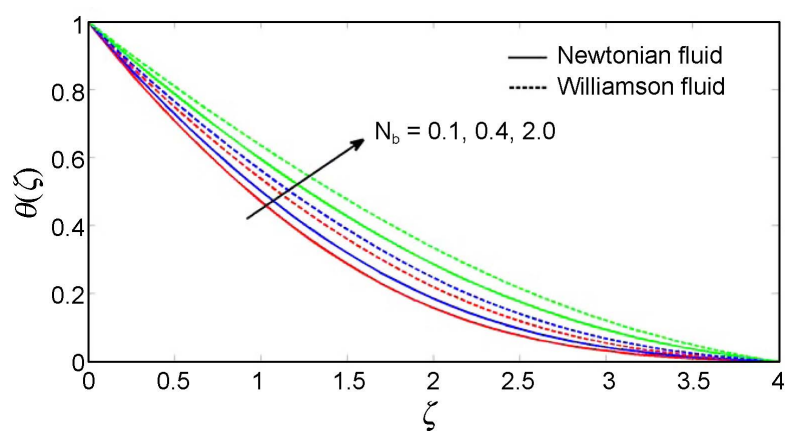

Fig. 3. (Color online) Temperature profile for different values of $N_{b}$ when $\Gamma=0.4, \operatorname{Pr}=1, N_{r}=0.5, \alpha=-1.15, \gamma=0.5, N_{t}=$ $0.2, L_{e}=0.5$.

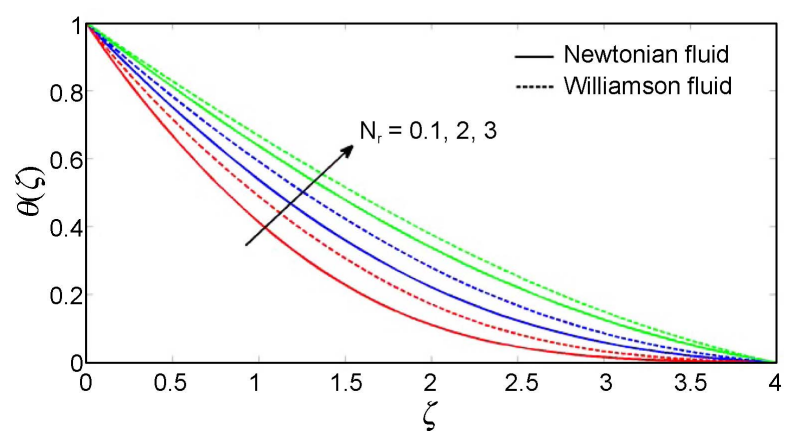

Fig. 4. (Color online) Temperature profile for different values of $N_{r}$ when $\Gamma=0.4, \operatorname{Pr}=1, \alpha=-1.15, \gamma=0.5, N_{b}=0.2, N_{t}=$ $0.2, L_{e}=0.5$.

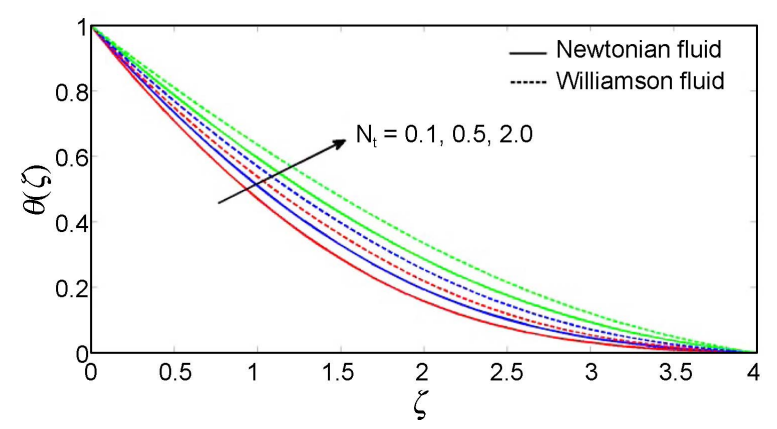

Fig. 5. (Color online) Temperature profile for different values of $N_{t}$ when $\Gamma=0.4, \operatorname{Pr}=1, N_{r}=0.5, \alpha=-1.15, \gamma=0.5, N_{b}=$ $0.2, L_{e}=0.5$.

of local Nusselt number and local Sherwood number for all the physical parameters. Figure 2 shows the velocity profile against magnetic and porosity parameter $(\Gamma)$. In this figure, we can observe that due to the increment in $(\Gamma)$, then the fluid velocity increases whereas when the fluid behavior depicts non-Newtonian then the fluid velocity diminish. The impact of Brownian motion parameter $\left(N_{b}\right)$ is displayed in Fig. 3. From this figure, we can analyze that temperature profile enhances due to the greater impact of Brownian motion parameter $\left(N_{b}\right)$. It can 


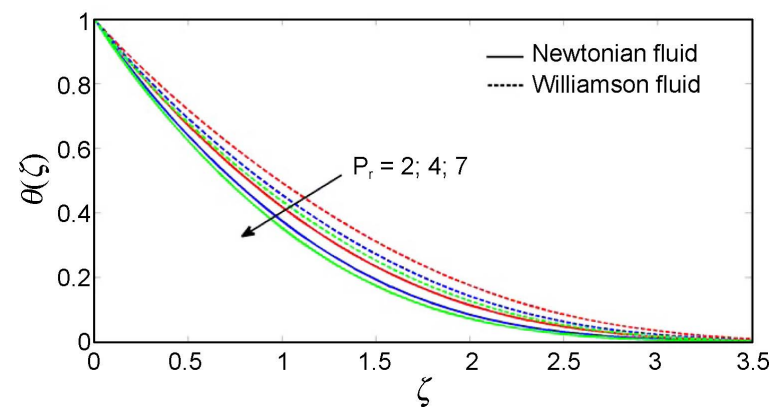

Fig. 6. (Color online) Temperature profile for different values of $\mathrm{P}_{\mathrm{r}}$ when $\Gamma=0.4, N_{r}=0.5, \alpha=-1.15, \gamma=0.5, N_{b}=0.2, N_{t}=$ $0.2, L_{e}=0.5$.

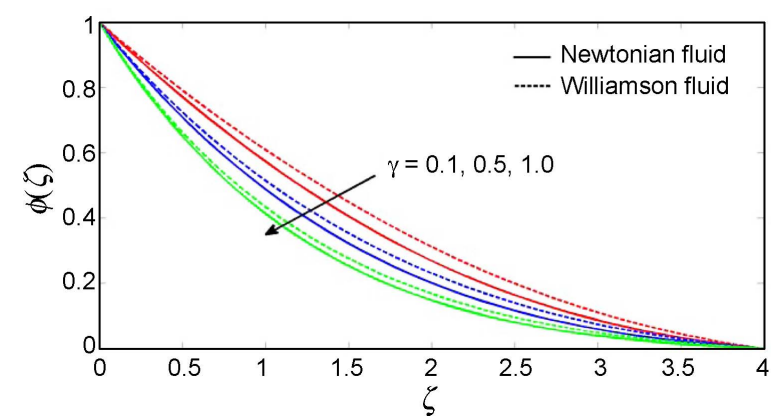

Fig. 7. (Color online) Concentration profile for different values of $\gamma$ when $\Gamma=0.4, \operatorname{Pr}=1, N_{r}=0.5, \alpha=-1.15, N_{b}=0.2$, $N_{t}=0.2, L_{e}=0.5$.

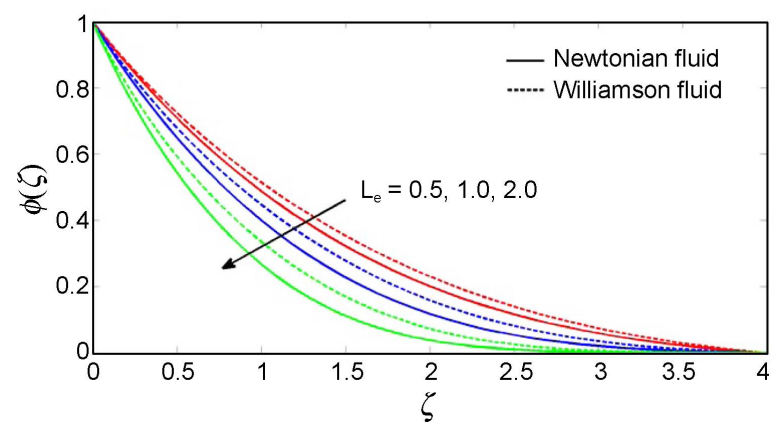

Fig. 8. (Color online) Concentration profile for different values of $L_{e}$ when $\Gamma=0.4, \operatorname{Pr}=1, N_{r}=0.5, \alpha=-1.15, \gamma=0.5$, $N_{b}=0.2, N_{t}=0.2$.

be illustrated by Fig. 4 that radiation parameter $\left(N_{r}\right)$ also enhances the temperature profile and boundary layer thickness. Larger values of radiation parameter $\left(N_{r}\right)$ gives more heat to the fluid which leads to increase the temperature profile and boundary layer thickness. Thermophoresis parameter $\left(N_{t}\right)$ also enhances the temperature profile as shown in Fig. 5, whereas the behavior of temperature profile becomes opposite for large values of Prandtl number $\left(P_{r}\right)$ as shown in Fig. 6. In other words, for greater values of Prandtl number $\left(P_{r}\right)$ leads to decrease

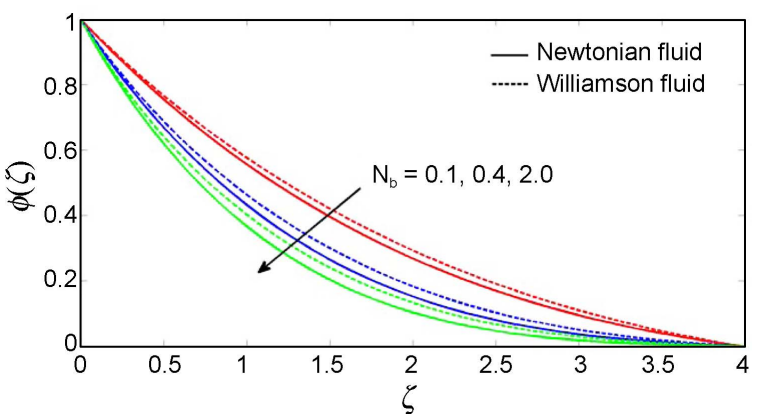

Fig. 9. (Color online) Concentration profile for different values of $N_{b}$ when $\Gamma=0.4, \operatorname{Pr}=1, N_{r}=0.5, \alpha=-1.15, \gamma=0.5, N_{t}$ $=0.2, L_{e}=0.5$.

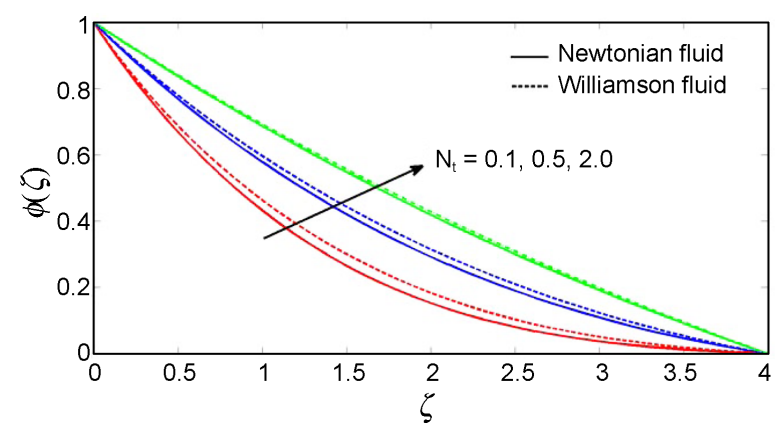

Fig. 10. (Color online) Concentration profile for different values of $N_{t}$ when $\Gamma=0.4, \operatorname{Pr}=1, N_{r}=0.5, \alpha=-1.15, \gamma=0.5, N_{b}$ $=0.2, L_{e}=0.5$.

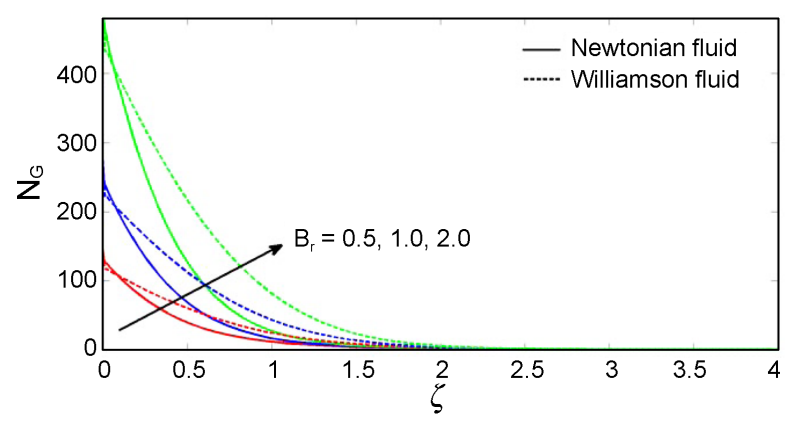

Fig. 11. (Color online) Entropy profile for different values of $B_{r}$ when $\Gamma=0.4, \operatorname{Pr}=1, N_{r}=0.5, \alpha=-1.15, \gamma=0.5, N_{b}=0.2$, $N_{t}=0.2, L_{e}=0.5$.

the thermal boundary layer thickness and opposes in spreading the heat.

Figure 7 to Fig. 10 are sketched to analyze the influence of Brownian motion parameter, thermophoresis parameter, Lewis number and Chemical reaction parameter on the dimensionless nanoparticle concentration profile. In Fig. 7 , we noticed that chemical reaction parameter $(\gamma)$ tends to reduce the concentration profile. Figure 8 represents the variation of Lewis number $\left(L_{e}\right)$ nanoparticle concentration profile. From this figure, we observed that for large 


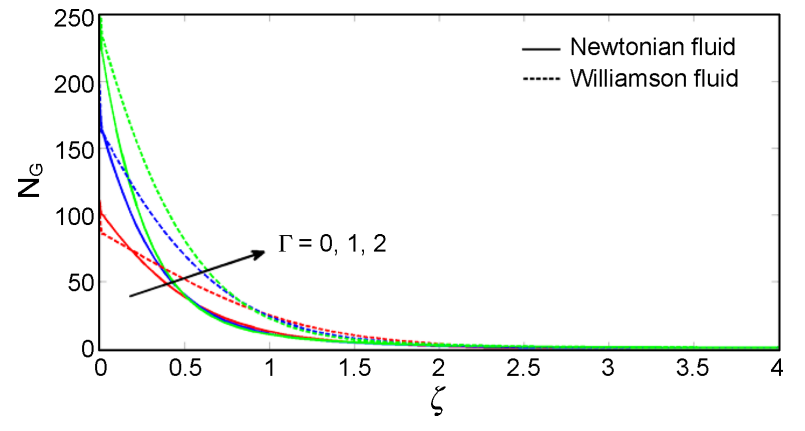

Fig. 12. (Color online) Entropy profile for different values of $\Gamma$ when $\operatorname{Pr}=1, N_{r}=0.5, \alpha=-1.15, \gamma=0.5, N_{b}=0.2, N_{t}=$ $0.2, L_{e}=0.5$.

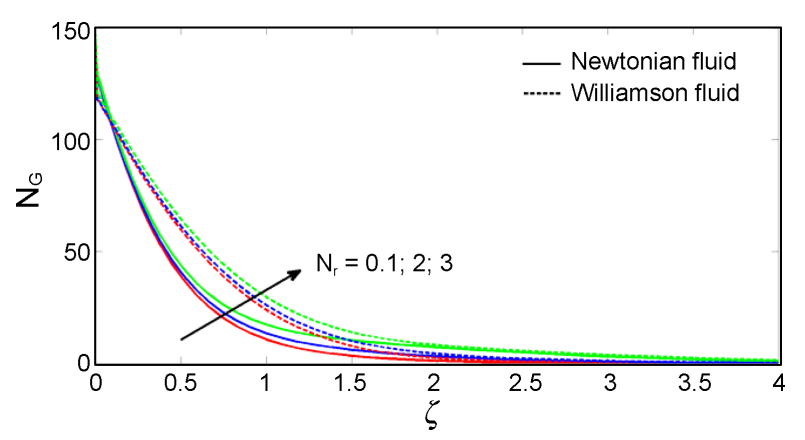

Fig. 13. (Color online) Entropy profile for different values of $N_{r}$ when $\Gamma=0.4, \operatorname{Pr}=1, \alpha=-1.15, \gamma=0.5, N_{b}=0.2, N_{t}=0.2$, $L_{e}=0.5$.

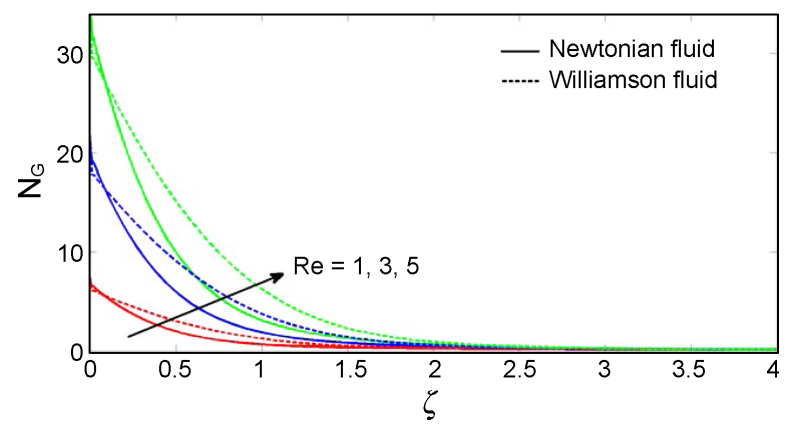

Fig. 14. (Color online) Entropy profile for different values of Re when $\Gamma=0.4, \operatorname{Pr}=1, N_{r}=0.5, \alpha=-1.15, \gamma=0.5, N_{b}=$ $0.2, N_{t}=0.2, L_{e}=0.5$.

values of Lewis number $\left(L_{e}\right)$ nanoparticle concentration profile diminish and its associated boundary layer thickness. In Fig. 9, we found that Brownian motion parameter $\left(N_{b}\right)$ causes a reduction in nanoparticle concentration profile. From Fig. 10, we observed that nanoparticle concentration profile and its associated boundary layer thickness increases due to the greater influence of thermophoresis parameter $\left(N_{t}\right)$. Entropy profiles are sketched in Fig. 11 to Fig. 14 for Brinkmann number, Reynolds number, Radiation parameter, magnetic and porosity parameter. In all these we observed that all the parameters enhance the entropy profile and if the fluid is non-Newtonian then entropy profile enhances more.

\section{Conclusion}

In this article, entropy generation on MHD Williamson nanofluid over a porous shrinking sheet has been analyzed under the influence of nonlinear thermal radiation and chemical reaction. The governing equation is transformed into nonlinear ordinary differential equation with the help of similarity transformations variables. The obtained resulting coupled equations are solved with the help of Successive linearization method and Chebyshev spectral collocation method. The major outcomes of the present analysis are summarized below:

- Velocity profile behaves as an increasing function for large values of $\Gamma$.

- Thermal radiation effects enhance the temperature profile, whereas Prandtl number shows opposite behavior.

- Entropy profile increases for all the physical parameters.

- An increment in chemical reaction parameter and Lewis number shows a decrement in nanoparticle concentration profile.

- Temperature profile and concentration profile increases for large values of thermophoresis parameter.

- The present analysis is also presented for Newtonian fluid by taking $\lambda=0$, as a special case of our study.

\section{Nomenclature}

$\tilde{u}, \tilde{v} \quad:$ Velocity components $(\mathrm{m} / \mathrm{s})$

$x, y \quad$ : Cartesian coordinate $(\mathrm{m})$

$\tilde{p} \quad$ : Pressure $\left(\mathrm{N} / \mathrm{m}^{2}\right)$

$\tilde{k} \quad$ : Porosity parameter

Re : Reynolds number

$N_{G} \quad$ : Dimensionless entropy number

Re : Reynolds number

$\tilde{t} \quad:$ Time (s)

$\mathrm{P}_{\mathrm{r}} \quad$ : Prandtl number

$\bar{k} \quad$ : Mean absorption coefficient

$S \quad:$ Suction/injection parameter

$N_{b} \quad$ : Brownian motion parameter

$N_{t} \quad$ : Thermophoresis parameter

$q_{w} \quad:$ Heat flux

$L_{e} \quad$ : Lewis number

$q_{m} \quad$ : Mass flux

$B_{r} \quad$ : Brinkman number

$T_{\infty} \quad$ : Environmental temperature (K)

$M \quad$ : Hartman number 
$B_{0} \quad$ : Magnetic field

$N_{r} \quad$ : Radiation parameter

$\tilde{T}, C$ : Temperature $(\mathrm{K})$ and Concentration

$g \quad:$ Acceleration due to gravity $\left(\mathrm{m} / \mathrm{s}^{2}\right)$

$\mathrm{D}_{\mathrm{B}} \quad$ : Brownian diffusion coefficient $\left(\mathrm{m} / \mathrm{s}^{2}\right)$

$D_{T} \quad$ : Thermophoretic diffusion coefficient $\left(\mathrm{m} / \mathrm{s}^{2}\right)$

$K \quad$ : Chemical reaction parameter

\section{Greek Symbol}

$\bar{\alpha} \quad$ : Thermal conductivity of the nano particles

$\alpha \quad$ : Stretching parameter

$\lambda \quad$ : Williamson fluid parameter

$\bar{\sigma} \quad:$ Stefan-Boltzmann constant

$\mu \quad$ : Viscosity of the fluid $\left(\mathrm{Ns} / \mathrm{m}^{2}\right)$

$\chi, \lambda_{1}$ : Dimensionless constant parameter

$\Omega \quad$ : Dimensionless temperature difference

$\phi \quad$ : Nanoparticle concentration

$\theta \quad:$ Temperature profile

$\sigma \quad$ : Electrical conductivity $(\mathrm{S} / \mathrm{m})$

$\varphi \quad$ : Stream function

$\tau \quad:$ Effective heat capacity of nano particle $(\mathrm{J} / \mathrm{K})$

$v \quad:$ Nano fluid kinematic viscosity $\left(\mathrm{m}^{2} / \mathrm{s}\right)$

$\gamma \quad$ : Dimensionless chemical reaction parameter

\section{References}

[1] S.U.S. Choi, ASME-Publications-Fed. 231, 99 (1995).

[2] Y. Xuan, and Q. Li, J. Heat Trans. 125, 151 (2003).

[3] J. Buongiorno, J. Heat Trans. 128, 240 (2006).

[4] W. A. Khan and I. Pop, Int. J. Heat Mass Transf. 53, 2477 (2010).

[5] M. Mustafa, T. Hayat, I. Pop, S. Asghar, and S. Obaidat, Int. J. Heat Mass Transf. 54, 5588 (2011).

[6] M. Hassan, R. Ellahi, and A. Zeeshan, Math. Sci. Lett. 5, 1 (2016).

[7] R. Ellahi, M. Hassan, and A. Zeeshan, Nanotech. IEEE Transac. 14, 726 (2015).

[8] M. M. Bhatti and M. M. Rashidi, J. Mol. Liq. 221, 567 (2016).

[9] M. Sheikholeslami, K. Vajravelu, and M. M. Rashidi, Int. J. Heat Mass Trans. 92, 339 (2016).

[10] A. Zeeshan, A. Majeed, and R. Ellahi, J. Mol. Liq. 215, 549 (2016).

[11] A. Bejan, CRC Press (1996).

[12] R. Ellahi, M. Hassan, and A. Zeeshan, Int. J. Heat Mass Trans. 81, 449 (2015).

[13] A. Zeeshan, M. Hassan, R. Ellahi, and M. Nawaz, P. I. Mech. Eng. E-J Pro. 0954408916646139 (2016).

[14] H. F. Oztop and K. Al-Salem, Renew. Sust. Energ. Rev.
16, 911 (2012)

[15] M. M. Rashidi, S. Abelman, and N. F. Mehr, Int. J. Heat Mass Transf. 62, 515 (2013).

[16] M. H. Abolbashari, N. Freidoonimehr, F. Nazari, and M. M. Rashidi, Powder Technol. 267, 256 (2014).

[17] M. H. Abolbashari, N. Freidoonimehr, F. Nazari, and M. M. Rashidi, Adv. Powder Technol. 26, 542 (2015).

[18] M. Sheikholeslami and D. D. Ganji, Physica A. 417, 273 (2015).

[19] M. Sheikholeslami and D. D. Ganji, Energy 75, 400 (2014).

[20] N. S. Akbar, Entropy. 17, 1411 (2015).

[21] M. Sheikholeslami, T. Hayat, and A. Alsaedi, Int. J. Heat Mass Trans. 96, 513 (2016).

[22] M. Sheikholeslami and S. Abelman, Nanotech. IEEE Transac. 14, 561 (2015).

[23] M. Sheikholeslami, M. M. Rashidi, and D. D. Ganji, J. Mol. Liq. 212, 117 (2015).

[24] M. Sheikholeslami, R. Ellahi, M. Hassan, and S. Soleimani, Int. J. Numer. Method. H. 24, 1906 (2014).

[25] Z. Abbas, M. Sheikh, and S. S. Motsa, Energy 95, 12 (2016).

[26] J. A. Khan, M. Mustafa, T. Hayat, and A. Alsaedi, Int. J. Heat Mass Transf. 86, 158 (2015).

[27] M. M. Rashidi, M. Ali, N. Freidoonimehr, B. Rostami, and M. A. Hossain, Adv. Mech. Eng. 6, 735939 (2014).

[28] M. M. Rashidi, N. Vishnu Ganesh, A. K. Abdul Hakeem, and B. Ganga, J. Mol. Liq. 198, 234 (2014).

[29] M. Sheikholeslami, M. M. Rashidi, and D. D. Ganji, Comput. Methods in Appl. Mech. Eng. 294, 299 (2015).

[30] M. S. Kandelousi, Eur. Phy. J. Pl. 129, 1 (2014).

[31] A. Zeeshan and A. Majeed, J. Magn. 21, 153 (2016).

[32] M. Sheikholeslami, T. Hayat, and A. Alsaedi, Int. J. Heat Mass Transf. 96, 513 (2016).

[33] M. Sheikholeslami, D. D. Ganji, and M. M. Rashidi, J. Taiwan Inst. Chem. Eng. 47, 6 (2015).

[34] M. Sheikholeslami, M. M. Rashidi, T. Hayat, and D. D. Ganji, J. Mol. Liq. 218, 393 (2016).

[35] M. S. Kandelousi, Phys. Lett. A. 378, 3331 (2014).

[36] M. Sheikholeslami, J. Braz. Soc. Mech. Sci. Eng. 37, 1623 (2015).

[37] M. M. Bhatti, T. Abbas, M. M. Rashidi, and M. E. S. Ali, Entropy. 18, 200 (2016).

[38] J. Qing, M. M. Bhatti, M. A. Abbas, M. M. Rashidi, and M. E. S. Ali, Entropy. 18, 123 (2016).

[39] M. M. Bhatti, T. Abbas, M. M. Rashidi, M. E. S. Ali, and Z. Yang, Entropy. 18, 224 (2016).

[40] M. Sheikholeslami, H. R. Ashorynejad, and P. Rana, J. Mol. Liq. 214, 86 (2016).

[41] M. M. Bhatti, A. Shahid, and M. M. Rashidi, Alexandria Eng. J. 55, 51 (2016). 